\title{
Site Dependent Oxidation and Reduction Mechanisms in Nanoparticles Investigated via Environmental Scanning Transmission Electron Microscopy
}

\author{
Alec P. LaGrow ${ }^{1 *}$, David C. Lloyd, ${ }^{2,3}$ Edward D. Boyes ${ }^{2,3,4}$, and Pratibha L. Gai ${ }^{2,3,5}$ \\ ${ }^{1 .}$ International Iberian Nanotechnology Laboratory, Braga, 4715-330, Portugal \\ 2.The York Nanocentre, The University of York, York, YO10 5DD, United Kingdom \\ ${ }^{3}$ Department of Physics, The University of York, York, YO10 5DD, United Kingdom \\ 4.Department of Electronics, The University of York, York, YO10 5DD, United Kingdom \\ ${ }^{5}$ Department of Chemistry, The University of York, York, YO10 5DD, United Kingdom \\ * Corresponding author: alec.lagrow@inl.int
}

Understanding the behavior of nanoparticles under reactive conditions is important to their use as heterogeneous catalysts, and in preventing corrosion. In-situ characterization techniques are a powerful way to probe these processes under simulated or actual reaction conditions. One such technique is environmental transmission electron microscopy (ETEM), which allows reactions to be studied under simulated reaction conditions with atomic resolution [1]. In ETEM gases are introduced into the column of the microscope directly, while the temperature is controlled with a heated micro-electromechanical holder. However, to follow phase changes in ETEM, typically atomic resolution is need which limits the overall area that can be studied in a single reaction. With the development of the environmental scanning transmission electron microscope (ESTEM) [2], atomic number (Z-contrast) sensitive imaging can be carried out using high angle annular dark field STEM (HAADF-STEM) imaging. HAADFESTEM allows phase transformations to be readily followed during oxidation and reduction in-situ by studying the changes in the Z-contrast associated with the changes in density of the nanomaterial $[3,4]$.

By tracking the changes in Z-contrast, large areas of nanoparticles can be studied simultaneously to follow oxidation and reduction mechanisms in nanoparticle systems. Here we discuss the oxidation processes in metal nanoparticles with a range of nanoparticle sizes and shapes and the reduction of their associated oxides. The oxidation and reduction reactions were studied in-situ on the double spherical aberration corrected Environmental (Scanning) TEM (AC-E(S)TEM) JEOL 2200FS at the University of York [5]. All nanoparticle systems were either pre-reduced in hydrogen at $500{ }^{\circ} \mathrm{C}$ in-situ [3,4], or heated to $350{ }^{\circ} \mathrm{C}$ under vacuum to remove surfactants before they were studied [6]. Subsequent oxidation or reduction experiments are carried out between $200{ }^{\circ} \mathrm{C}$ and $500{ }^{\circ} \mathrm{C}$ with a flow of oxygen or hydrogen gas giving a pressure at the sample, in the microscope, of $2 \mathrm{~Pa}$.

The use of metal nanoparticle systems and shape-controlled metal nanoparticles allow the oxidation mechanism to be followed from the area of initial nucleation, through to full oxidation. In the nanoparticle systems the oxidation occurs initially from the smallest corner of the metal, this occurs in cases where there is only a single nucleation event (i.e. with copper nanoparticles) [3]. In cases with multiple nucleation events and shelling of the particles, the oxidation starts from the corners of the particles in all cases, and then a polycrystalline shell grows to cover the entire particle [4]. Similar results are seen in the reduction of the associated metal oxides. If the nanoparticles are fully oxidized into hollow metal oxide structures, particle collapse is observed where smaller parts of the particles are ejected and the rest of the metal forms low energy nanoparticles [4]. During reduction the metal atoms migrate to the lowest energy surfaces often forming nanoparticle systems bound by their low energy facets or removing higher energy sites such as corners to regain lower energy configurations (Figure 1). 
In partially oxidized shape controlled systems, such as triangular plates of nickel, this reduction process rounds out the higher energy corners and increases the amount of material on the lower energy edges of the particle (Figure 1).

\section{References:}

[1] E. D. Boyes, and P. L. Gai, P. L. Ultramicroscopy 67, (1997), 219.

[2] E. D. Boyes, M.R. Ward, L. Lari, and P. L. Gai, Annalen Der Physik 525, (2013), 423.

[3] A. P. LaGrow et al., Journal of the American Chemical Society 139 (2017), 179.

[4] A. P. LaGrow, D. C. Lloyd, P. L. Gai, and E. D. Boyes, Chemistry of Materials 30, (2018), 197.

[5] P. L. Gai, E. D. Boyes, Microscopy Research Technology. 72, (2009), 153.

[6] A. P. LaGrow et al., Journal of Microscopy. 269, (2018), 161.

[7] A grant from the EPSRC (UK) supporting this work is acknowledges. The authors thank the research grant EP/J0118058/1.
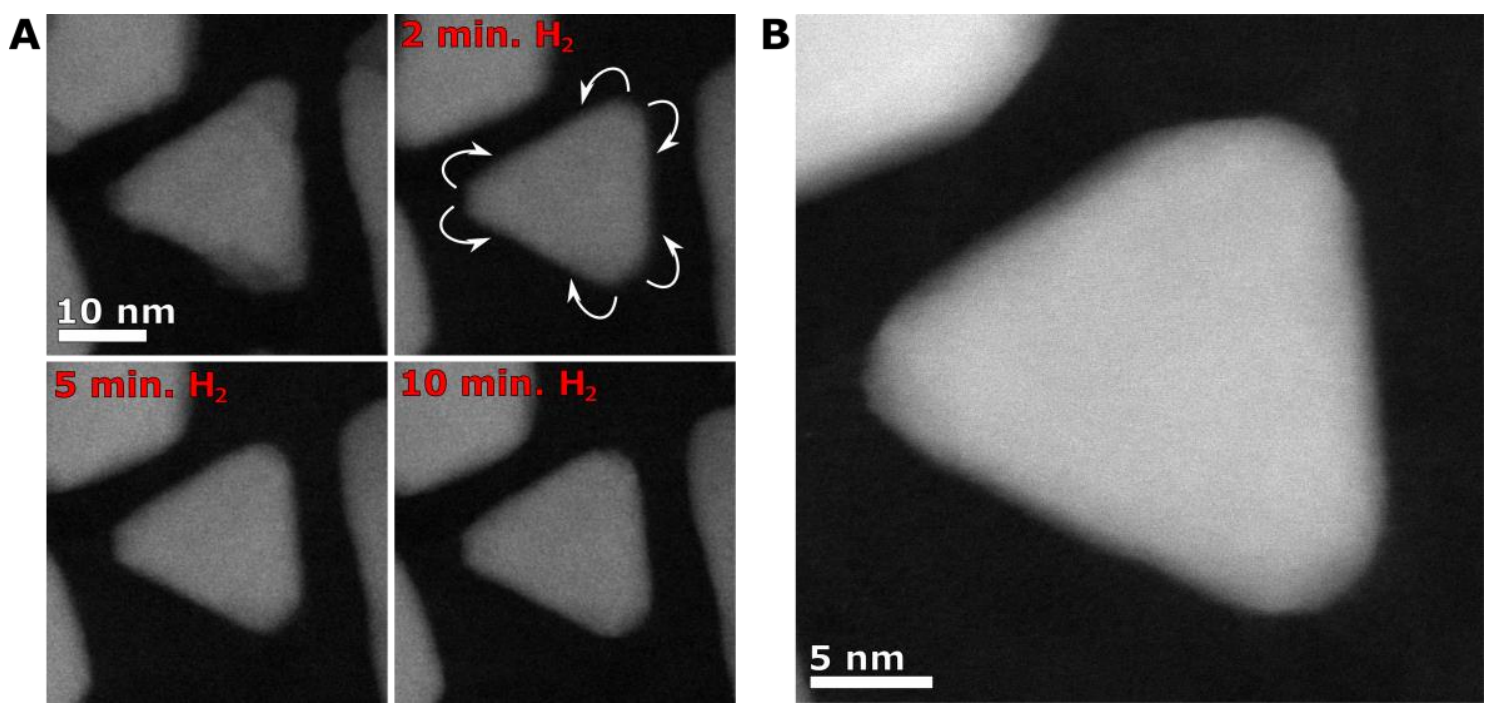

Figure 1. In-situ visualization of dynamic reduction of triangular nanoplates with their corners preferentially oxidized in HAADF-ESTEM. A) The reduction of the oxide corners over the course of 10 minutes in hydrogen with the areas showing the migration of material away from the corners. B) A higher magnification image of the triangular plate after reduction with the higher energy corners rounded. 